\title{
Correction to: A newly developed APCC SCOPS and its prediction of East Asia seasonal climate variability
}

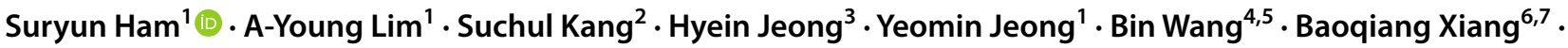 \\ Shu $\mathrm{Wu}^{8}$. Joshua Xiouhua $\mathrm{Fu}^{9}$
}

Published online: 2 August 2019

(c) Springer-Verlag GmbH Germany, part of Springer Nature 2019

\section{Correction to: Climate Dynamics https://doi.org/10.1007/s00382-018-4516-5}

Upon publication, it came to our attention that certain revisions were necessary to honor the agreement between University of Hawaii and APEC Climate Center for the project, "Development of APCC Seamless Prediction System". There are three sections that are to be corrected to accurately reflect the agreement of project.

First, we would like inform that four authors, Bin Wang, Baoqiang Xiang, Shu Wu, and Joshua Xiouhua Fu, were inadvertently omitted from the first published version of our manuscript. The revised authorship has been updated to the following list:

Suryun Ham, A-Young Lim, Suchul Kang, Hyein Jeong, Yeomin Jeong, Bin Wang, Baoqiang Xiang, Shu Wu, and Joshua Xiouhua Fu

Second, in the original publication of the article, the acknowledgement has been published incorrectly. The correct acknowledgement should be:

The original article can be found online at https://doi.org/10.1007/ s00382-018-4516-5.

Suryun Ham

suryun01@apcc21.org

1 Climate Services and Research Department, APEC Climate Center, Busan 48058, South Korea

2 Ralph M. Parsons Laboratory, Massachusetts Institute of Technology, Cambridge, MA, USA

3 Fluid Dynamics and Solid Mechanics (T-3), Theoretical Division, Los Alamos National Laboratory, Los Alamos, NM, USA

4 Department of Atmospheric Sciences and International Pacific Research Center, School of Ocean and Earth Science and Technology, University of Hawaii, Hawaii, USA
"This research was supported by the APEC Climate Center. Also, this study was supported by the Korea Meteorological Administration. We especially thank KMA's supercomputer management division for providing us with the supercomputer resource and consulting on technical support. Some of ocean data were collected and made freely available by the International Argo Program and the national programs that contribute to it. (http://www.argo.ucsd.edu, http:// argo.jcommops.org). The Argo Program is part of the Global Ocean Observing System. Also, this research is based on APCC Project (2015), "Development of APCC Seamless Prediction System" by APCC with a research group of the University of Hawaii, USA. Dr. Bin Wang acting as PI of the project leads and directs the APCC project (2012-2015). Dr. Baoqiang Xiang develops the atmosphere-ocean-sea ice coupled model system. Dr. Shu Wu develops the EAKF initialization and observational data pre-process package. Dr. Joshua Xiouhua Fu develops the 3D-nudging atmosphere initialization".

Lastly, in the original publication of the article, one sentence in the section $2 \mathrm{~b}$ should be revised. The original sentence read as follows:

5 Earth System Modeling Center, Nanjing, China

6 NOAA/Geophysical Fluid Dynamics Laboratory, Princeton, NJ, USA

7 University Corporation for Atmospheric Research, Boulder, CO, USA

8 Nelson Institute Center for Climatic Research, University of Wisconsin-Madison, Madison, WI, USA

9 International Pacific Research Center, School of Ocean and Earth Science and Technology, University of Hawaii, Hawaii, USA 
"Based on the POEM system, SCoPS has been newly developed as a fully coupled climate model for seamless prediction of weather and climate (APCC project report 2015)"

The correct sentence should be:

"Based on the POEM system, a research group at University of Hawaii developed the original version of the in-house prediction model for APCC under the "Agreement between the APEC Climate center and the University of Hawaii on the APCC international research project for development of APCC seamless prediction system (APCC project report 2015)".
We would like inform that none of these errors affect our previously published results.

Publisher's Note Springer Nature remains neutral with regard to jurisdictional claims in published maps and institutional affiliations. 